Review

\title{
Oligodendrogliomas: Clinicopathological correlations
}

\author{
Johan M. Kros \\ Department of Pathology/Neuropathology Laboratory, University Hospital Rotterdam-Dijkzigt, Dr. Molewa- \\ terplein 40,3015 GD Rotterdam, The Netherlands
}

Key words: brain tumor, glioma, oligodendroglioma, outcome, proliferation, histopathology

Since the original description of glial neoplasms consisting of cells with oligodendroglial morphology [1] many papers have addressed the clinical behavior of this tumor. While their name was derived from a characteristic appearance in silver stains as tumors composed of glial cells with few and short processes, the hallmark of oligodendrogliomas as shown in hematoxylin and eosin stains became the typical honeycomb structure. Soon after its recognition it became clear that in a considerable number of oligodendrogliomas astrocytic cells were encountered, and after the introduction of immunohistochemical stains for glial fibrillary acidic protein, a marker for the astrocytic cell lineage, even more oligodendroglial neoplasms appeared to harbor astrocytic cells $[2,3]$. Only a limited number of recent studies specifically addresses the problem of how to distinguish oligodendrogliomas from the mixed oligo-astrocytomas by mentioning maximum allowable percentages of astrocytic cells [6-9]. In recent years immunohistochemical markers specifically reacting with the cells of oligodendrogliomas have been sought but not yet found $[4,5]$.

Besides the well known difficulties in predicting clinical outcome of neoplasms based on histopathology, as interference of other prognostically relevant parameters, and besides the limited representation of a small biopsy sample from tumors as heterogenous as gliomas [10], clinicopathological investigations were hampered by the relatively rareness of this member of the glioma family. Furthermore, there is no consensus regarding the legitimation of individual histopathological features used in grading schemes for oligodendrogliomas. In several recent studies grading schemes consisting of various features have been tested with regard to their predictive power $[6,7,9,11,12]$. No major differences were apparent between the survival curves resulting from application of the original, rather illdefined scheme of Kernohan, and those obtained by using the schemes later developed by the AFIP, or that used by a french-american group (St. AnneMayo) $[9,12]$.

Some studies were aimed at identifying individual histopathological features with significance for prognosis. The results of these studies do not provide a consensus. In a large Norwegian study the features cell density, necrosis and microcysts were found to have prognostic significance [7]. In contrast, in the largest study on oligodendrogliomas to date so far, viz. the AFIP series, only nuclear pleomorphism was identified as individual significant factor [6]. Stepwise regression analysis used in another American study revealed that necrosis and the number of mitoses contained all prognostically usefull information. The St. Anne-Mayo scheme, not exclusively used for oligodendrogliomas, but successfully applied to astrocytomas as well [13], promised to become a good scheme in grading oligodendrogliomas because of encompassing features of undebatable significance [12]. In addition to cell density, nuclear pleomorphism, endothelial abnormality and necrosis, the feature of mitotic index, proven to be a significant parameter, is used in this scheme as well $[9,11]$. However, application on a group of cases of the Mayo Clinic yielded no more 
than two (and not three) distinct survival curves [12].

Immunohistochemical analysis of proliferation using anti-Ki-67 antibody including that developed for formalin-fixed, paraffin-embedded tissue Mib-1, yielded good correlations with tumor grade and clinical outcome [14]. Ploidy patterns obtained by DNA-flow cytometry on oligodendroglial tumors were not correlated with histopathological features, nor indicative for biological behavior [15]. However, S-phase fraction roughly correlated with tumor grade [10].

The role of age in clinical outcome of patients suffering from oligodendrogliomas has been a matter of controversy. In the large study by Mørk patient's age seemed not to influence survival [7]. On the contrary, age was found to be an important prognostic factor correlating with tumor grade in several other studies $[3,6,11,12,16]$. In our own material we found that younger patients survive longer. However, confounding factors in age effects on survival are correlations of younger age with lower histopathological grade and with more favorable (i.e., frontal) localizations of the oligodendrogliomas [16].

While inconclusive and contradictory statements concerning the influence of tumor localization were made in the earliest reports on oligodendrogliomas [17-19], only few recent studies focussed attention on tumor site [12,16]. In 1950 Earnest and Kernohan wrote that tumor site, i.e., frontal versus non-frontal (hemispheric) localization, would provide all prognostic information, and that even grading the tumors in a two-tiered scheme (viz. oligodendrogliomas and oligodendroblastomas) would be redundant [19]. While the beneficial effect of frontal tumor site on survival could be confirmed indeed, the supposed irrelevance of grading was falsified by succesfully grading in the group of frontal localized neoplasms [16].

Satisfactory volumetric measurements, compatible with histopathologic tumor borders, can be obtained from CT scans. Estimations of tumor growth rate based on increase in tumor volume measured from subsequential CT scans, however, are limited to cases with relatively substantial changes in tumor bulk. In those 43 cases from our series in which CT scans were available, no correlation between tumor size and survival, nor between tumor size and histopathological grade, was found [16]. The results were not different for those patients who had undergone a stereotactic biopsy with assumed minimal removal of tumor tissue [16]. Tumor recurrence is supposed to be highly dependent on the residual tumor volume after surgery. The reliability of estimations of debulked tumor volume is questionable and in only one paper linked with survival [12]. Papers addressing the relation between volume of rest tumor and recurrence are missing because in many centers post-operative scans are not routinely made, and delineation of true tumor tissue from reactive changes based on CT images is difficult, if not impossible.

The effects of radiation therapy on oligodendrogliomas is still controversial, since all studies evaluating radiotherapy were done retrospectively without controlling for variables in patients or the tumors $[12,16,20,21-24]$. Analogous with the prospective, randomized trials in which it was found that high grade oligodendrogliomas in particular are responsive to chemotherapy $[25,26]$, trials for the effects of radiation therapy should be developed.

\section{References}

1. Bailey P, Hiller F: The interstitial tissue of the nervous system; a review. J Nerv \& Ment Dis 59: 337, 1924

2. Herpes MJHM, Budka $\mathrm{H}$ : Glial fibrillary acidic protein (GFAP) in oligodendroglial tumors: Gliofibrillary oligodendroglioma and transitional oligo-astrocytoma as subtypes of oligodendroglioma. Acta Neuropathol (Berl.) 64: 265-272, 1984

3. Wilkinson IMS, Anderson JR, Holmes AE: Oligodendroglioma: an analysis of 42 cases. J Neurol Neurosurg Psychiatry 50: 304-312, 1987

4. Nakagawa Y, Perentés E, Rubinstein LJ: Immunohistochemical characterization of oligodendrogliomas: an analysis of multiple markers. Acta Neuropathol (Berl.) 72:15-22, 1986

5. De la Monte SM: Uniform lineage of oligodendrogliomas. Am J Pathol 135(3): 529-540, 1989

6. Smith MT, Ludwig CL, Godfrey AD, Armbrustmacher VW: Grading of oligodendrogliomas. Cancer 52: 2107-2114, 1983

7. Mørk SJ, Halvorsen TB, Lindegaard K-F, Eide GE: Oligodendroglioma. Histologic evaluation and prognosis. $\mathrm{J}$ Neuropathol Exp Neurol 45(1): 65-78, 1986 
8. Sun ZM, Genka S, Shitara N, Akanuma A, Takakura K: Factors possibly influencing the prognosis of oligodendroglioma. Neurosurgery 22(5): 886-891, 1988

9. Kros JM, Troost D, van Eden CG, van der Werf AJM, Uylings HBM: Oligodendroglioma. A comparison of two grading systems. Cancer 61: 2251-2259, 1988

10. Coons SW, Johnson PC: Regional heterogeneity in the DNA content of human gliomas. Cancer 72: 3052-3060, 1993

11. Burger PC, Rawlings CE, Cox EB, McLendon RE, Schold SC, Bullard DE: Clinicopathologic correlations in the oligodendroglioma. Cancer 59: 1345-1352, 1987

12. Shaw EG, Scheithauer BW, O'Fallon JR, Tazelaar HD, Davis DH: Oligodendrogliomas: the Mayo Clinic experience. J Neurosurg 76: 428-434, 1992

13. Daumas-Duport C, Scheithauer BW, O'Fallon J, Kelly P: Grading of astrocytomas. A simple and reproducible method. Cancer 62: 2152-2165, 1988

14. Karamitopoulou E, Perentes E, Diamantis I, Maraziotis T: Ki-67 immunoreactivity in human central nervous system tumors: a study with MIB 1 monoclonal antibody on archival material. Acta Neuropathol (Berl.) 87: 47-54, 1994

15. Kros JM, van Eden CG, Vissers CJ, Mulder AH, van der Kwast ThH: Prognostic relevance of DNA flow cytometry in the oligodendroglioma. Cancer 69(7): 1791-1798, 1992

16. Kros JM, Pieterman H, van Eden CG, Avezaat CJJ: Oligodendroglioma: The Rotterdam-Dijkzigt Experience. Neurosurgery 34(6): 959-966, 1994

17. Martin JP: Two cases of oligodendroglioma with remarks on the general clinical features of such cases. Brain 54: 330-349, 1931

18. Greenfield JG, Robertson EG: Cystic oligodendrogliomas of the cerebral hemispheres and ventricular oligodendrogliomas. Brain 56: 247-264, 1933
19. Earnest F III, Kernohan JW, Craig WM: Oligodendrogliomas. A review of two hundred cases. Arch Neurol Psychiatry $63: 964-976,1950$

20. Chin HW, Hazel JJ, Kim TH, Webster JH: Oligodendrogliomas. 1. A clinical study of cerebral oligodendrogliomas. Cancer 45: 1458-1466, 1980

21. Reedy DP, Bay JW, Hahn JF: Role of radiation therapy in the treatment of cerebral oligodendroglioma: an analysis of 57 cases and a literature review. Neurosurgery 13(5): 499503,1983

22. Bullard DE, Rawlings CE, Phillips B, Cox EB, Schold SC, Burger PC, Halperin EC: Oligodendroglioma. An analysis of the value of radiation therapy. Cancer 60: 2179-2188, 1987

23. Lindegaard K-F, Mørk SJ, Eide GE, Halvorsen TB, Hatlevoll R, Solgaard T, Dahl O, Ganz J: Statistical analysis of clinicopathological features, radiotherapy, and survival in 170 cases of oligodendroglioma. J Neurosurg 67: 224-230, 1987

24. Wallner KE, Gonzales M, Sheline GE: Treatment of oligodendrogliomas with or without postoperative irradiation. $J$ Neurosurg 68: 684-688, 1988

25. Cairncross JG, Macdonald DR: successful chemotherapy for recurrent malignant oligodendroglioma. Ann Neurol 23: $360-364,1988$

26. Glass J, Hochberg FH, Gruber ML, Louis DN, Smith D, Rattner B: The treatment of oligodendrogliomas and mixed oligodendroglioma-astrocytomas with PVC chemotherapy. J Neurosurg 76: 741-745, 1992

Address for offprints: J.M. Kros, Department of Pathology/Neuropathology Laboratory, University Hospital Rotterdam-Dijkzigt, Dr. Molewaterplein 40, 3015 GD Rotterdam, The Netherlands 